\title{
FLOW ROUTING AIMING LOAD BALANCING AND PATH LENGTH IN MULTI-HOP NETWORKS WITH DIFFERENT LINK QUALITIES
}

\author{
Leizer L. Pinto*, Kátia C. C. Fernandes and Kleber V. Cardoso
}

\begin{abstract}
This paper considers an exact bi-objective approach for simultaneously minimizing the total cost of flow routing and the network bottleneck when link qualities and flow weights are relevant. This is useful for wireless multi-hop networks with long-term TCP (Transmission Control Protocol) flows. The introduced proposal can generate a minimal complete set of Pareto-optimal solutions. Our proposal is evaluated through simulation in which are explored different parameter settings and metrics.
\end{abstract}

Mathematics Subject Classification. 90C10, 90C29, 90C35, 90C90.

Received December 17, 2020. Accepted August 14, 2021.

\section{INTRODUCTION}

Wireless multi-hop networks, which is common to have different link qualities, have been widely investigated $[1,2]$. However, recently, this topic has started rising attention again due to the large adoption. For example, wireless mesh networks are becoming common in companies and households, while wireless sensors networks and vehicular networks are important building block for the smart cities [11,14]. In these networks, routing is essential for efficient utilization of the available capacity by distributing the traffic through multiple paths while taking into consideration the quality of the links $[9,10]$. From the applications perspective, taking into account the flows in the routing improves the performance, since reordering is avoided. Flow routing is specially useful for TCP (Transmission Control Protocol) flows, which are still dominant in the present and in the foreseeable future when video streaming exceeds $80 \%$ of all Internet traffic [7].

Due to the complexity, heuristic approaches have been the frequent choice for routing in multi-hop wireless networks with different link qualities [6,9]. However, there are some relevant theoretical works based on exact solutions. For example, Berman et al. [4], Martins [12], Gadegaard et al. [8] and Bornstein et al. [5] present exact algorithms for bi-objective problems in networks with cost and bottleneck functions, but in these works each network link $(i, j)$ has a value $b_{i j}$ associated with the bottleneck function. Thus, the bottleneck value of a solution $x$ is the greatest $b_{i j}$ of the links that compose $x$. Pinto et al. [15] also present an exact algorithm for a bi-objective network problem with cost and bottleneck functions, but different of $[4,5,8,12]$ the values assigned to the bottleneck function are not input data. In [15], these values depend on the number of flows to be routed on the links. However, the proposal in [15] does not take into consideration links with different qualities and flows with different weights.

Keywords. Network flow routing, bi-objective optimization, wireless multi-hop networks, load balancing, total path length.

Instituto de Informática (INF), Universidade Federal de Goiás (UFG), Goiânia, GO, Brazil.

* Corresponding author: leizer@inf.ufg.br 
In this paper, we revisit the bi-objective formulation, but dealing with networks that have links with different qualities. The link quality affects the effective capacity of the link. Thus, the flow weight on a link is also affected by the link quality. For example, as commented in [16], a path with low bandwidth can have a large weight while a path with large bandwidth can have a low weight. Additionally, we assume the network traffic is dominated by TCP-like flows, i.e., flows that are affected by the path length. As it is well-known, the longer the path of the TCP flow is, the longer is the feedback loop and smaller is the average throughput. As a consequence, the path length of a flow also affects its weight on a link.

Given the links qualities and the flows weights, the approach presented in [15] is not able to solve the problem described above. In order to deal with the different qualities associated with the links, we design a bottleneck function composed of two metrics: the link quality and the network flow weight. In Section 2, we introduce our proposed solution in detail and also describe the problem formulation. In Section 3, we present the evaluation of our proposal through simulation. We present our final considerations in Section 4.

\section{Problem formulation AND SOlution}

We consider routing in multi-hop networks in which the links can have different qualities. The network is represented by an oriented connected graph $G=(V, E)$, where $V$ represents the set of nodes (i.e., communication devices), with $|V|=n$, and $E$ the set of edges (i.e., links), with $|E|=m$. There is a set of flows $F$, with $|F|=r$, which must be routed in $G=(V, E)$. Each flow $f \in F$ must be routed through only one path, starting from the source $s^{f} \in V$ and ending in the destination $d^{f} \in V$. For each edge $(i, j) \in E$, from $i \in V$ to $j \in V$, and flow $f \in F$, we define a binary variable $x_{i j}^{f}$ that indicates whether or not the flow $f$ goes through the edge $(i, j)$. There is also a non-negative $\operatorname{cost} c_{i j}^{f}$ associated with the flow $f \in F$ while going through the edge $(i, j) \in E$.

Let $q_{i j} \in \mathbb{Z}_{\geq 0}$ be the quality of the edge $(i, j) \in E$, with $q_{i j} \in\left\{q_{1}, q_{2}, \cdots, q_{\xi}\right\}$ and $q_{1}<q_{2}<\cdots<q_{\xi}$, where $q_{1}$ represents the best quality and $q_{\xi}$ represents the worst quality. Consider $p^{f} \in \mathbb{Z}_{\geq 0}$ as the weight of flow $f \in F$. Thus, let $w_{i j}^{f}$ be the weighted sum of $p^{f}$ and $q_{i j}$, which is given by $w_{i j}^{f}=\beta_{1} \cdot p^{f}+\beta_{2} . q_{i j}, \forall f \in F$ and $\forall(i, j) \in E$, where the values $\beta_{1}, \beta_{2} \in \mathbb{Z}_{\geq 0}$ are parameters that allow to control the degree of importance of $p^{f}$ and $q_{i j}$, respectively. Thus, we formulate the bi-objective integer programming model to this problem, denoted by $(P)$, as follows:

$$
\begin{array}{r}
\operatorname{minimize}\left\{\max _{(i, j) \in E}\left\{\sum_{f \in F} w_{i j}^{f} \cdot x_{i j}^{f}\right\}\right\} \\
\operatorname{minimize}\left\{\sum_{f \in F(i, j) \in E} \sum_{i j} c_{i j}^{f} \cdot x_{i j}^{f}\right\}
\end{array}
$$

subject to:

$$
\begin{gathered}
\sum_{(i, j) \in E} x_{i j}^{f}-\sum_{(j, i) \in E} x_{j i}^{f}=\left\{\begin{array}{ll}
1, & \text { if } i=s^{f} \\
0, & \forall i \in V-\left\{d^{f}, s^{f}\right\}, \\
-1, & \text { if } i=d^{f}
\end{array} \quad \forall f \in F\right. \\
x_{i j}^{f} \in\{0,1\}, \forall(i, j) \in E \text { and } \forall f \in F .
\end{gathered}
$$

The network bottleneck represents the load in the edge(s) carrying the proportional largest amount of traffic and is given by $z_{1}(x)=\max _{(i, j) \in E}\left\{\sum_{f \in F} w_{i j}^{f} \cdot x_{i j}^{f}\right\}$. The total cost of all flows, i.e., the sum of the lengths of the paths 
of all flows, is given by $z_{2}(x)=\sum_{f \in F} \sum_{(i, j) \in E} c_{i j}^{f} \cdot x_{i j}^{f}$. In general the objective functions (2.1) and (2.2) may be conflicting, since minimizing the network bottleneck may result in increasing the sum of the path lengths. The constraints ensure that each flow $f \in F$ leaves the source $s^{f}$ and arrives at the destination $d^{f}$, going through exactly one single path. We present some additional relevant definitions in the following.

Let $X$ be the set of all feasible solutions of $(P)$, and let $z(x)=\left[z_{1}(x), z_{2}(x)\right]$ be the objective vector that is associated with $x \in X$. Given two solutions $x, x^{\prime} \in X$, the solution $x$ dominates $x^{\prime}$ if $z(x) \leq z\left(x^{\prime}\right)$ and $z(x) \neq z\left(x^{\prime}\right)$, where the operators $\leq$ and $\neq$ regard vector comparisons. Thus, a feasible solution $x \in X$ is a Pareto-optimal solution if there is no other solution in $X$ that dominates $x$. The set $X^{*} \subseteq X$, composed of Pareto-optimal solutions, is a minimal complete set if: 1) for any $x^{*}, \bar{x} \in X^{*}$ we have $z\left(x^{*}\right) \neq z(\bar{x})$; and, 2) for any Pareto-optimal solution $x \in X$ there exists $x^{*} \in X^{*}$ such that $z(x)=z\left(x^{*}\right)$.

In order to solve the problem $(P)$, we have adapted the algorithm proposed in [15], as presented in the following:

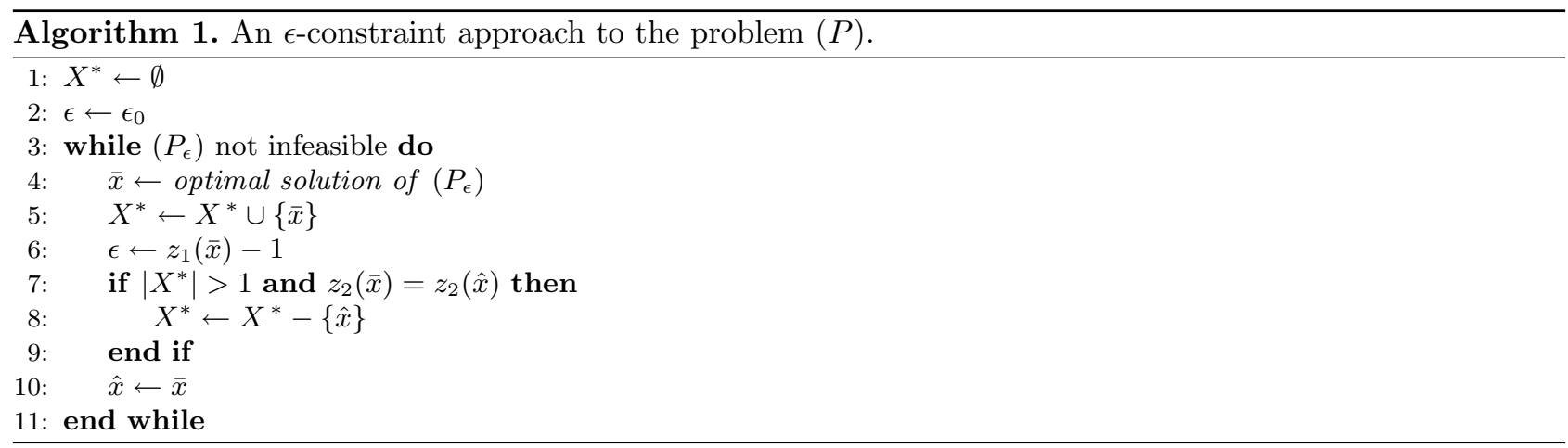

$\left(P_{\epsilon}\right)$ is a single-objective zero-one linear programming subproblem which the objective function is $(2.2)$ of $(P)$. The constraints of $(P)$ remain in $\left(P_{\epsilon}\right)$. Additionally, the objective function $(2.1)$ of $(P)$ is turned into a constraint in $\left(P_{\epsilon}\right)$, as follows:

$$
\sum_{f \in F} w_{i j}^{f} \cdot x_{i j}^{f} \leq \epsilon, \forall(i, j) \in E .
$$

The Algorithm (1) solves $\left(P_{\epsilon}\right)$ repeatedly, employing an updated value of $\epsilon$ in each iteration. As the qualities of the edges and the weights of flows are taken into account in $z_{1}$, then the largest bottleneck occurs when all flows cross an edge with the worst quality, which we denote as the maximum value $\epsilon_{0}$. Thus, we start $\epsilon=\epsilon_{0}=\sum_{f \in F}\left[\beta_{1} \cdot p^{f}+\beta_{2} \cdot q_{\xi}\right]$, line 2 of Algorithm (1). After obtaining the optimal solution $\bar{x}$ to $\left(P_{\epsilon}\right)$, we update $\epsilon=z_{1}(\bar{x})-1$. This makes the bottleneck of the new solution $\bar{x}$ smaller than the previous one $\hat{x}$. If the value of $z_{2}$ is the same for these two solutions, the previous solution is dominated by the new one and it must be deleted. For any feasible solution $x$ for the problem $(P), z_{1}(x) \in \mathbb{Z}_{\geq 0}$, since the values of $w_{i j}^{f}$ and $x_{i j}^{f}$ are nonnegative integers. Therefore, there is no feasible solution with $z_{1}$ greater than $z_{1}(\bar{x})-1$ and less than $z_{1}(\bar{x})$. This ensures that, at the end of Algorithm (1), it generates a minimal complete set of Pareto-optimal solutions $X^{*}$ to $(P)($ see $[5,15])$.

Note that the minimum value of $\epsilon$ is $\epsilon_{f}=\beta_{1} \cdot \bar{p}+\beta_{2} \cdot q_{1}$, where $\bar{p}=\max _{f \in F}\left\{p^{f}\right\}$, i.e., $\epsilon_{f}$ is the minimum possible value to the bottleneck objective function. As $\epsilon$ decreases by at least one unit in each iteration, $\left(P_{\epsilon}\right)$ is solved at most for $\hat{\epsilon}$ iterations, where $\hat{\epsilon}=\epsilon_{0}-\epsilon_{f}+1$. It follows that the cardinality of $X^{*}$ is limited by $\hat{\epsilon}$, i.e., $\left|X^{*}\right| \leq \hat{\epsilon}$. Therefore, the complexity of Algorithm (1) is $O(\hat{\epsilon} . g(\epsilon))$, where $g(\epsilon)$ is the complexity of $\left(P_{\epsilon}\right)$. 


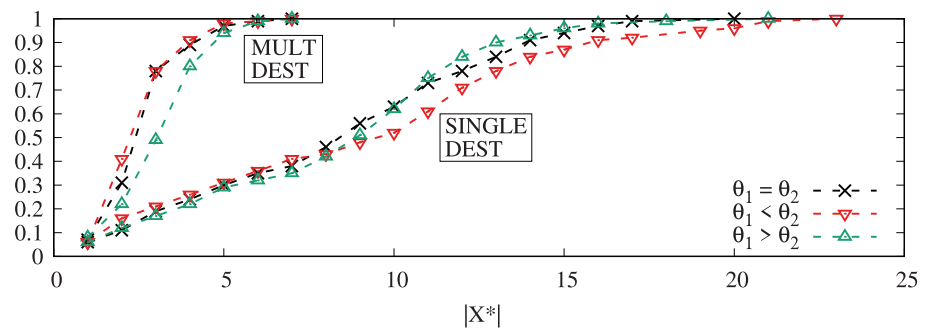

Figure 1. Cardinality of the minimal complete set of Pareto-optimal solutions.

\section{Performance evaluation}

In order to evaluate the proposal, we have implemented a custom simulator in $\mathrm{C}++$ language. This approach facilitates the integration with the CPLEX optimization solver through the Concert library and the extraction of different metrics related to both the algorithm and the quality of the solutions. We employed a randomly generated network topology with 100 nodes. This topology was generated by the Barabási-Albert model [3], as described in the following. The network starts with $\bar{n}_{0}$ nodes. Each new node establishes a connection with $\bar{n}$ previously added nodes, such that $\bar{n} \leq \bar{n}_{0}$. The probability $p_{i}$ that a new node will be connected to a node $i$ depends on its connectivity $k_{i}$, so that $p_{i}=k_{i} / \sum_{j \in V} k_{j}$. Our topology uses $\bar{n}=\bar{n}_{0}=3$ and $c_{i j}^{f}=1, \forall f \in F$ and $\forall(i, j) \in E$.

As previously stated, the link quality affects the effective capacity of the link and, consequently, the flow weight over a link is also affected by the link quality. Thus, in order to represent an estimation of the relative weight of each flow, we consider $p^{f}=\left[\gamma / \Gamma^{f}\right]$, where $\Gamma^{f}$ is the shortest path length of $f \in F$ and $\gamma=\max _{f \in F}\left\{\Gamma^{f}\right\}$. The flows were randomly distributed according to two settings: (1) multiple sources and multiple destinations, and (2) multiple sources and a single destination. In each setting, we evaluated two scenarios, one with 40 flows and other with 80 flows, employing 100 simulation runs for each one.

One out three possible values, representing the link qualities, was randomly assigned to each edge. In other words, $q_{i j} \in\left\{q_{1}, q_{2}, q_{3}\right\}$, i.e., $\xi=3$. We made $\beta_{1}=\left\lceil\theta_{1} \cdot \frac{q_{\xi}}{\hat{p}}\right\rceil$, turning $\theta_{1}$ into a parameter with the same dimension of $\beta_{2}$. For the sake of clarity, we use $\beta_{2}=\theta_{2}$. We evaluated $\theta_{1}$ and $\theta_{2}$ with the following values: $\left(\theta_{1} ; \theta_{2}\right)=(1 ; 1),(1 ; 10)$, and $(10 ; 1)$.

The evaluation of our proposal is based on three metrics: (i) the cardinality of the minimal complete set of Pareto-optimal solutions $\left(\left|X^{*}\right|\right)$, (ii) the number of iterations of $(P)$ in which $\left(P_{\epsilon}\right)$ was solved, and (iii) the execution time. The evaluation of the quality of the Pareto-optimal solutions returned by our new algorithm is based on the relative decrease in the bottleneck. This metric is computed as the ratio between the last Paretooptimal solution and the first Pareto-optimal solution generated by the algorithm. The ratio is computed over only one vector dimension which corresponds to the bottleneck.

Figure 1 presents the cumulative distribution function $(\mathrm{CDF})$ of the cardinality of the minimal complete set of Pareto-optimal solutions with 40 flows. In the setting in which the flows have multiple destinations (and multiple sources), these flows tend to be spread along the network and the load tends to be distributed over several edges. As a consequence, there are few Pareto-optimal solutions. In the setting in which the flows have single destination (and multiple sources), there is high load concentrated over edges with end-point in the sink node. Since there is a large number of edges with end-point in the sink node, this implies on larger cardinality of the minimal complete set of Pareto-optimal solutions, which represent the several non-dominated solutions considering both objectives the bottleneck and the total number of hops. The difference in the ratio between $\theta_{1}$ and $\theta_{2}$ has small impact. The results for 80 flows are not presented here, but the general behavior is similar, naturally, with higher values. 


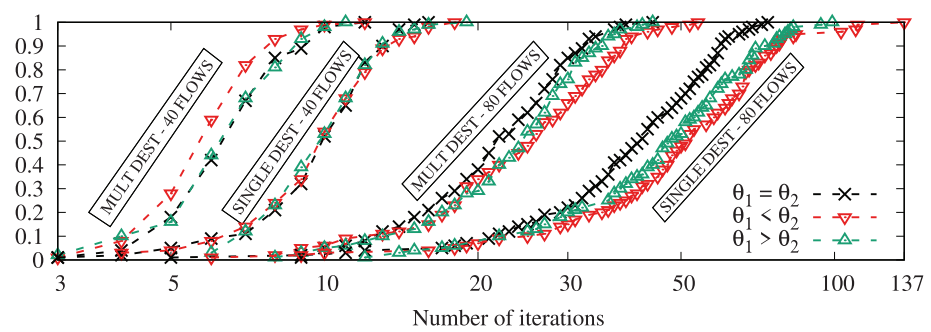

FiguRE 2. Number of iterations in which $\left(P_{\epsilon}\right)$ was solved.

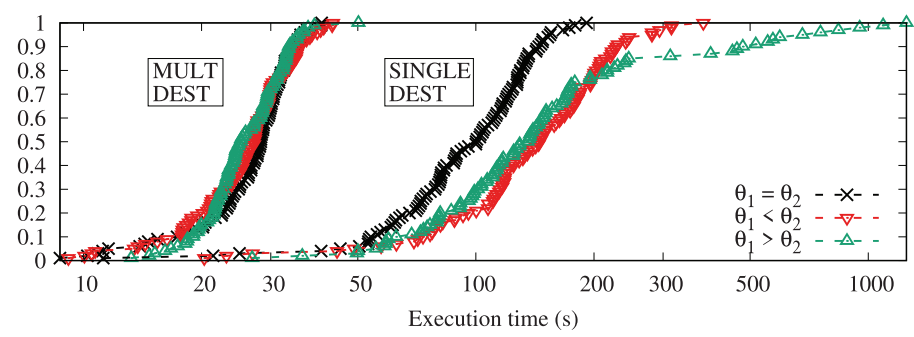

FIgURE 3. Execution time in seconds.

Figure 2 shows the CDF of the number of iterations in which $\left(P_{\epsilon}\right)$ was solved. Given the results related to the cardinality of $X^{*}$, we already expected the difference between multiple destinations and single destination concerning the number of iterations of $(P)$. The difference between the number of flows was also expected and it is clearly illustrated in this figure. The impact of the difference in the ratio $\theta_{1}$ and $\theta_{2}$ remains small. However, some consistent impact can be noticed with 80 flows and single destination. The number of iterations tend to be higher when $\theta_{1}$ differs from $\theta_{2}$.

Figure 3 presents the CDF of the execution time (in seconds) with 80 flows. The difference in the number of iterations of the algorithm between multiple destinations and single destination has influence in the execution time of these settings. However, when $\theta_{1}>\theta_{2}$, there are situations that demand for evaluating a large number of combinations for the generated solutions. The results for 40 flows (not presented here) allows differentiation between multiple destinations and single destination, but not between the ratios involving $\theta_{1}$ and $\theta_{2}$ (similarly to the results of the number of iterations).

Figures $4 \mathrm{a}$ and $4 \mathrm{~b}$ present the relative decrease in the bottleneck with 80 flows. While the maximum decrease in both settings, multiple destinations and single destination, is similar, the overall CDFs are very different. In the setting of single destination, all scenarios involve a similar set of links that converge to the sink node. Thus, the difference among the scenarios (i.e., the CDF inclination) comes from the random position of the sources and the destination. In the setting of multiple destinations, the bottlenecks are spread and the distribution of bottleneck decrease is strongly influenced by the topology. In this topology generated by Barabási-Albert model, most of the decrease is between $30 \%$ and $50 \%$. In tests with a grid topology, under the same conditions, we observed a different CDF of the bottleneck decrease. In the setting of multiple destinations, becomes clear the difference between $\theta_{1}>\theta_{2}$ and other two ratios. As expected, with this parametrization, the bottleneck decrease is the lowest. However, $\theta_{1}=\theta_{2}$ and $\theta_{1}<\theta_{2}$ have very similar behavior. The results for 40 flows are not presented here, but the general behavior is similar. 


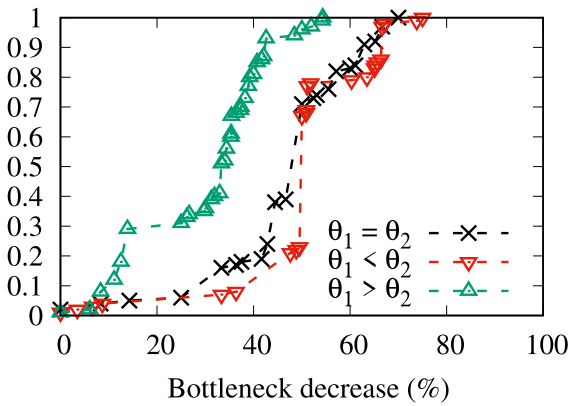

(a)

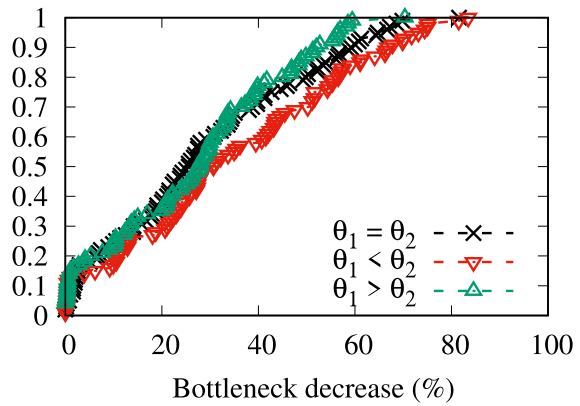

(b)

FIGURE 4. Percentage of the relative decrease in the bottleneck. (a) Multiple destinations; (b) Single destination.

\section{Conclusion and Final Remarks}

In this work, based on the $\epsilon$-constraint approach, we introduced a flow routing proposal that takes into account the link qualities and the flow weights. This exact approach is able to generate a minimal complete set of Pareto-optimal solutions. Through simulation, we evaluate how this approach can be used to route flows whose the performance is affected by the bottleneck and also by the path length, such as long-term TCP flows. As future work, we intend to improve further our model in order to capture the stochastic behavior of wireless multi-hop networks.

Acknowledgements. The authors are grateful to Prof. Ridha Mahjoub and two anonymous reviewers of this journal for their helpful comments and suggestions for improving the paper.

\section{REFERENCES}

[1] I.F. Akyildiz, W. Su, Y. Sankarasubramaniam, and E. Cayirci, Wireless sensor networks: a survey. Comput. Netw. 38 (2002) 393-422.

[2] I.F. Akyildiz, X. Wang, and W. Wang, Wireless mesh networks: a survey. Comput. Netw. 47 (2005) 445-487.

[3] A.-L. Barabási and R. Albert, Emergence of scaling in random networks. Science 286 (1999) 509-512.

[4] O. Berman, D. Einav, and G. Handler, The constrained bottleneck problem in networks. Oper. Res. 38 (1990) $178-181$.

[5] C.T. Bornstein, N. Maculan, M. Pascoal, and L.L. Pinto, Multiobjective combinatorial optimization problems with a cost and several bottleneck objective functions: An algorithm with reoptimization. Comput. Oper. Res. 39 (2012) $1969-1976$.

[6] M.O. de Mello, V.C. Borges, L.L. Pinto, and K.V. Cardoso, Improving load balancing, path length, and stability in low-cost wireless backhauls. Ad Hoc Netw. 48 (2016) 16-28.

[7] Q. Fan, H. Yin, G. Min, P. Yang, Y. Luo, Y. Lyu, H. Huang, and L. Jiao, Video delivery networks: Challenges, solutions and future directions. Comput. Electr. Eng. 66 (2018) 332-341.

[8] S.L. Gadegaard, A.Klose, and L.R. Nielsen, A bi-objective approach to discrete cost-bottleneck location problems. Ann. Oper. Res. 267 (2016) 179-201.

[9] J.J. Gálvez and P.M. Ruiz, Efficient rate allocation, routing and channel assignment in wireless mesh networks supporting dynamic traffic flows. Ad Hoc Netw. 11 (2013) 1765-1781.

[10] Q. Liu, J. Yin, V.C.M. Leung, and Z. Cai, ISAR: improved situation-aware routing method for wireless mesh backbones. IEEE Commun. Lett. 16 (2012) 1404-1407.

[11] W. Lu, Y. Gong, X. Liu, J. Wu, and H. Peng, Collaborative energy and information transfer in green wireless sensor networks for smart cities. IEEE Trans. Industr. Inform. 4 (2018) 1585-1593.

[12] E.Q.V. Martins, On a special class of bicriterion path problems. Euro. J. Oper. Res. 17 (1984) 85-94.

[13] R. Martin, M. Menth, and M. Hemmkeppler, Accuracy and dynamics of hash-based load balancing algorithms for multipath internet routing. In: 2006 3rd International Conference on Broadband Communications, Networks and Systems. IEEE (2006) pp. 1-10.

[14] P. Patil, A. Hakiri, Y. Barve, and A. Gokhale, Enabling Software-Defined Networking for Wireless Mesh Networks in smart environments. In: IEEE 15th International Symposium on Network Computing and Applications (NCA). IEEE (2016) $153-157$. 
[15] L.L. Pinto, K.C.C. Fernandes, K.V. Cardoso, and N. Maculan, An Exact and polynomial approach for a bi-objective integer programming problem regarding network flow routing. Compute. Oper. Res. 106 (2019) 28-35.

[16] S. Prabhavat, H. Nishiyama, N. Ansari, and N. Kato, On load distribution over multipath networks. IEEE Commun. Surv. Tutor. 14 (2011) 662-680.

\section{Subscribe to Open (S2O) A fair and sustainable open access model}

This journal is currently published in open access under a Subscribe-to-Open model (S2O). S2O is a transformative model that aims to move subscription journals to open access. Open access is the free, immediate, online availability of research articles combined with the rights to use these articles fully in the digital environment. We are thankful to our subscribers and sponsors for making it possible to publish this journal in open access, free of charge for authors.

\section{Please help to maintain this journal in open access!}

Check that your library subscribes to the journal, or make a personal donation to the $\mathrm{S} 2 \mathrm{O}$ programme, by contacting subscribers@edpsciences.org

More information, including a list of sponsors and a financial transparency report, available at: https://www. edpsciences.org/en/maths-s2o-programme 